\title{
A Survey on Sentiment Classification for Product Aspect Ranking
}

\author{
Neha M. Toshniwal \\ ME Scholar (Computer Engineering) \\ PESMCOE, Pune.
}

\author{
D.V. Gore \\ Assistant Professor (Computer Engineering) \\ PESMCOE, Pune.
}

\begin{abstract}
A large number of reviews for the product are available on the internet .To classify these reviews is very difficult task. The Sentiment classification is one of the ongoing research areas in text mining field which is used for classifying the polarity of the reviews. In this paper, we study the survey of different techniques for sentiment classification.
\end{abstract}

\section{General Terms}

Aspects, online customer reviews, sentiment analysis

\section{Keywords}

Sentiment analysis, sentiment classification, product reviews.

\section{INTRODUCTION}

Sentiment classification (or Opinion mining) is the study of the people opinion, attitudes and emotions. Sentiment classification extracts the subjective information from the reviews and helps the customers and the manufacturers to improve the product features. Sentiment analysis helps to classify the polarity of the aspects as positive, negative or neutral. Sentiment analysis is carried out on the document level, sentence level or aspect level.

In document level the whole document is classified as positive or negative class. In sentence level sentiment classification classifies sentences as positive, negative, or neutral. And in the aspect level we identify and extract the features from the source data.

There are various machine learning algorithms for sentiment classification which are used to classify the text. This paper presents a survey on the different techniques used for classification of the sentiments.

\section{SENTIMENT ANALYSIS TECHNIQUES}

There are various machine learning approaches in which mostly used are the supervised classification methods. Machine learning techniques like Naïve Bayes (NB), Maximum Entropy (ME), and Support Vector Machine (SVM) are widely used.

In machine learning technique the training dataset is collected first and classifier is trained on the training data and the feature selection is done on the basis of the term present and the term frequency, once the classification technique is used.

Zha et. al. [1] categorized the positive and negative opinions on the aspects from the pros and cons of the reviews. Here the classifier is trained by the Pros and cons reviews, which is used to determine customer opinion on the aspects in free text reviews. They compared the three supervised classifiers SVM, NB, ME in terms of F1Measure, which is a combination of precision and recall.
Here the SVM was implemented using the libSVM, NB with Laplace smoothing and ME was implemented with LBFGS parameter estimation. The experiment are conducted on the 21-products in eight domains found that the SVM performs better than NB and ME.

D.K.Kirange et. al. [2] conducted experiments on the laptop and the restaurant reviews dataset and compared the performance of the system with the $\mathrm{KNN}(\mathrm{K}$ - Nearest Neighbor) and SVM, and compared their accuracy for sentiment classification, and concluded that the SVM performs better than KNN .

M.GOVINDARAJAN [3] found that there are the disadvantages for the SVM classifier as its performance reduces for the small dataset. So proposed a new approach which is the assemble of classifier NB and SVM known as Hybrid approach, which is applied for the sentiment classification tasks, with the aim of efficiently integrating the advantages of the NB and SVM. The proposed approach is based on 5 phases and the experiments were conducted on the $10 * 10$ fold cross validation for evaluating the accuracy on the dataset of 2000 movie reviews. And compared the results with the NB and SVM and concluded that the proposed approach NBSVM gives higher accuracy than the base classifiers (NB and SVM).

Nguyen et. al. [4] introduced a study combining the advantages of the both NB and SVM classifier into a two stage system by applying the reject option for the document-level sentiment classification. Here the NB classifier was used in the first stage and the documents rejected by the NB where given as input to the second stage classifier SVM and the classification is done. The experiments were conduct on publicly available standard polarity dataset of movie reviews and evaluate the classifier based on 10 fold cross validation. Results were obtained without reject option and with reject option. SVM gains higher accuracy without reject option and NBSVM gains higher accuracy with reject option.

Wang and manning [5] showed that the inclusion of the word bigram features gives consistent gain on sentiment analysis. They presented a simple model where SVM is built over NB log-count ratios as feature values. The experiments were carried on the different publicly available dataset on different methods and found that the NBSVM is the robust performer than other classifiers and the benefits of the bigrams depend on the dataset used.

Kim et. al. [7] proposed a simple but novel approach to adjust the term frequency portion in tf-idf, which is an unsupervised weighting scheme, by assigning credibility adjusted score to each tokens. The comparison is done against traditional tf-idf weighting scheme on multiple benchmarks and the method proposed gives better results on multiple benchmarks, which included both the snippets and longer documents. 
D. Tang et. al. [8] proposed a method, in which the problem of polarity inconsistency in the pipelined methods was overcome. The method proposed is joint segmentation and classification framework for sentiment analysis, which simultaneously conducts the sentence segmentation and sentence level sentiment classification. The experiments results were conducted on the Twitter dataset in SemEval 2013 and shows that the joint model outperforms the pipelined methods.

Wouter Bancken et. al. [9] proposed and domain independent approach for aspect based sentiment analysis to obtain most positive and negative aspects of a specific product from the free text customer reviews. The aspects are identified by the handcraft dependency paths in individual sentence. This approach does not require the seed word or domain specific knowledge as it employs offthe-shelf sentiment lexicon. The experiments were conducted on the on-line movie reviews and the MP3 player reviews and the accuracy of the top $n$ aspects were calculated.

The following table 1 .Shows the different methods and the performance parameter used for the different datasets.

Table 1. Performance parameter and the dataset used for different methods.

\begin{tabular}{|c|c|c|c|c|}
\hline $\begin{array}{l}\text { Sr } \\
\text { no. }\end{array}$ & Paper & $\begin{array}{l}\text { Classification } \\
\text { method }\end{array}$ & Dataset used & Performance Parameter \\
\hline 1 & $\begin{array}{l}\text { Product Aspect Ranking And its } \\
\text { Applications[1] }\end{array}$ & SVM & $\begin{array}{l}\text { Cnet.com, viewpoint } \\
\text { s.com in } 8 \text { domains }\end{array}$ & F-Measure \\
\hline 2 & $\begin{array}{l}\text { A Two-Stage Classifier for Sentiment } \\
\text { Analysis[2] }\end{array}$ & NBSVM & Movie reviews & Reject rate accuracy \\
\hline 3 & $\begin{array}{l}\text { Credibility Adjusted Term } \\
\text { Frequency[3] }\end{array}$ & $\begin{array}{l}\text { cred-tf-idf and } \\
\text { NBSVM }\end{array}$ & $\begin{array}{l}\text { Short and long } \\
\text { movie reviews }\end{array}$ & Tf-idf \\
\hline 4 & Emotion classification[4] & $\mathrm{KNN}$ & $\begin{array}{l}\text { Restaurant and } \\
\text { laptop reviews }\end{array}$ & Accuracy \\
\hline 5 & $\begin{array}{l}\text { Sentiment Classification using Hybrid } \\
\text { Method[5] }\end{array}$ & $\begin{array}{l}\text { Hybrid } \\
\text { NBSVM }\end{array}$ & 2000 movie reviews & $\begin{array}{l}\text { Cross validation } \\
\text { technique, accuracy }\end{array}$ \\
\hline 6 & $\begin{array}{l}\text { Performance of Hybrid Sentiment } \\
\text { Classification[6] }\end{array}$ & $\begin{array}{l}\text { Hybrid } \\
\text { classifier SVM }\end{array}$ & $\begin{array}{l}\text { Online customer } \\
\text { reviews }\end{array}$ & Misclassification rate \\
\hline 7 & $\begin{array}{l}\text { Sentiment Categorization with Machine } \\
\text { Learning Techniques[7] }\end{array}$ & Adaboost & Movie reviews & 3 fold classification \\
\hline 8 & $\begin{array}{l}\text { A Joint Segmentation and } \\
\text { Classification Framework[8] }\end{array}$ & JSC & Twitter data & F-measure \\
\hline 9 & $\begin{array}{l}\text { Baselines and Bigrams: Simple, Good } \\
\text { Sentiment[9] }\end{array}$ & NBSVM & IMDB, RTs, CR & $\begin{array}{l}\text { log count ratio, } 10 \text {-fold } \\
\text { cross validation }\end{array}$ \\
\hline 10 & $\begin{array}{l}\text { Automatically Detecting and Rating } \\
\text { Product Aspects from Textual } \\
\text { Customer Reviews[10] }\end{array}$ & Aspectator & $\begin{array}{l}\text { Online movie \& } \\
\text { mp3 player reviews }\end{array}$ & Accuracy \\
\hline
\end{tabular}

From the table 1 it is concluded that the performance of the classification method used depends on the method and dataset used.

The performance parameters are calculated as given below:

Accuracy is calculated as:

Accuracy $=\operatorname{sum}($ abs $($ Expected output - actual output $)) / 2$.

F1-measure is a combination of precision and recall, as

$\mathrm{F} 1=2 *($ precision $*$ recall $/($ precision + recall $))$

Precision $=$ number of true positive instances/ total no. of positive instances.

Recall $=$ no. of positive instances/total no. instances.

Misclassification rate $=(\mathrm{C} 1+\mathrm{C} 2) /$ total no. of reviews

Where, $\mathrm{C} 1=$ negative reviews classified as positive.

$\mathrm{C} 2=$ positive reviews classified as negative.

\section{CONCLUSION}

The different methods for the sentiment classification such as SVM, Hybrid methods, Aspectator, JSC are studied in brief, and come to conclude that the performance of the method used depends on the dataset and the methods used for classification. In future more improved methods can be used for sentiment classification to improve the performance of the system.

\section{REFERENCES}

[1] Z-J-Zha, J-Yu, J-tang, M-wang, T-S Chua Product aspect ranking and its applications ", IEEE Transaction of Knowledge and Data Engineering, Vol.26, no.5, may-2014.

[2] Dai Quoc Nguyen and Dat Quoc Nguyen and Son Bao Pham," A Two-Stage Classifier for Sentiment Analysis, International Joint Conference on Natural Language Processing", pages 897-901, Nagoya, Japan, 14-18 October 2013.

[3] Yoon Kim, Owen Zhang,"Credibility Adjusted Term Frequency: A Supervised Term Weighting Scheme for Sentiment Analysis and Text Classification", June 2014.

[4] D. K. Kirange, Ratnadeep R. Deshmukh," Emotion Classification of Restaurant and Laptop Review Dataset: SemEval 2014 Task 4 “, International Journal of Computer Applications (0975 - 8887) Volume 113 - No. 6, March 2015.

[5] M.GOVINDARAJAN,“SENTIMENT CLASSIFICATION OF MOVIE REVIEWS USING HYBRID METHOD", International Journal of Advances in Science Engineering and Technology, ISSN: 2321-9009 Volume- 1, Issue-3, Jan.-2014.

[6] G.Vinodhini et al. "Performance of Hybrid Sentiment Classification Model on Online Product Reviews". International Journal of Computer Science 
Engineering (IJCSE), ISSN : 2319-7323 Vol. 2 No.06. Nov 2013

[7] Andr'es Cassinelli, Chih-Wei Chen," CS 224N Final Project Boost up! Sentiment Categorization with Machine Learning Techniques”, June 5, 2009.

[8] Duyu Tang, Furu Wei, Bing Qin , Li Dong, Ting Liu , Ming Zhou, "A Joint Segmentation and Classification Framework for Sentiment Analysis", Proceedings of the 2014 Conference on Empirical Methods in Natural Language Processing (EMNLP), pages 477-487, October 25-29, 2014, Doha, Qatar. c 2014 Association for Computational Linguistics.

[9] Sida Wang and Christopher D.Manning, "Baselines and Bigrams: Simple, Good Sentiment and Topic Classification", Department of Computer Science, Stanford University, Stanford, CA 94305

[10] Wouter Bancken, Daniele Alfarone and Jesse Davis, "Automatically Detecting and Rating Product Aspects from Textual Customer Reviews", Proceedings of
DMNLP, Workshop at ECML/PKDD, Nancy, France, 2014.

[11] B. Liu, Sentiment Analysis and Opinion Mining .Morgan \& Claypool Publishers, San Rafael, CA, USA, 2012.

[12] B. Pang, L. Lee, and S. Vaithyanathan, "Thumbs up? Sentiment classification using machine learning techniques," in Proc. EMNLP, Philadelphia, PA USA, 2002, pp. 79-86.

[13] J. Yu, Z.-J. Zha, M. Wang, and T. S. Chua, "Aspect ranking: Identifying important product aspects from online consumer reviews," in Proc. ACL, Portland, OR, USA, 2011, pp. 1496-1505.

[14] L. M. Manevitz and M. Yousef, "One-class SVMs for document classification," J. Mach. Learn., vol. 2, pp. 139-154, Dec. 2011.

[15] M. Hui and B. Liu, "Mining andSummarizing customer reviews," in Proc. SIGKDD, Seattle, WA, USA, 2004, pp. 168-177. 